\title{
Spin-preserving ultrafast carrier capture and relaxation in InGaAs quantum dots
}

Cite as: Appl. Phys. Lett. 87, 153113 (2005); https://doi.org/10.1063/1.2103399

Submitted: 09 May 2005 . Accepted: 23 August 2005 . Published Online: 07 October 2005

S. Trumm, M. Wesseli, H. J. Krenner, D. Schuh, M. Bichler, J. J. Finley, and M. Betz

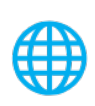

\section{ARTICLES YOU MAY BE INTERESTED IN}

Independent dynamic acousto-mechanical and electrostatic control of individual quantum dots in a LiNbOz-GaAs hybrid

Applied Physics Letters 106, 013107 (2015); https://doi.org/10.1063/1.4905477

Surface acoustic wave regulated single photon emission from a coupled quantum dotnanocavity system

Applied Physics Letters 109, 033105 (2016); https://doi.org/10.1063/1.4959079

Surface acoustic wave modulation of a coherently driven quantum dot in a pillar microcavity Applied Physics Letters 111, 011103 (2017); https://doi.org/10.1063/1.4990966
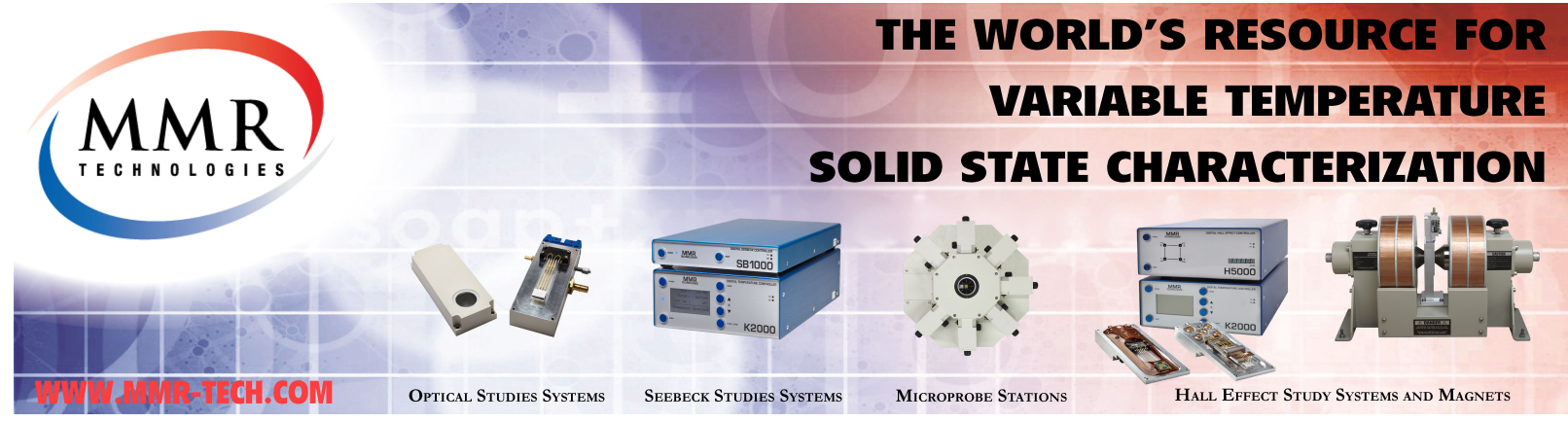


\title{
Spin-preserving ultrafast carrier capture and relaxation in InGaAs quantum dots
}

\author{
S. Trumm and M. Wesseli \\ Physik-Department E 11, Technische Universität München, James-Franck-Str., \\ D-85748 Garching, Germany \\ H. J. Krenner, D. Schuh, M. Bichler, and J. J. Finley \\ Walter-Schottky-Institut and Physik-Department E24, Technische Universität München, Am Coulombwall, \\ D-85748 Garching, Germany \\ M. Betz $\left.{ }^{a}\right)$ \\ Physik-Department E11, Technische Universität München, James-Franck-Str., D-85748 Garching, Germany
}

(Received 9 May 2005; accepted 23 August 2005; published online 7 October 2005)

Carrier capture into self-organized InGaAs/GaAs quantum dots with an electronic level spacing close to optical phonon energies is studied in a two-color femtosecond transmission experiment. After resonant generation of carriers in the wetting layer, we analyze the population of both the band edge of the wetting layer and the excited states of the quantum dots. Most strikingly, the carrier capture time of $3 \mathrm{ps}$ is found to be independent of the carrier density, providing that it remains small compared to the number of available electronic states in the quantum dots. Moreover, we find that the capture process is predominantly spin preserving in nature. These results suggest that phonon-mediated scattering governs the quantum dot filling. (C) 2005 American Institute of Physics.

[DOI: $10.1063 / 1.2103399]$

Semiconductor quantum dots (QDs) are currently attracting much attention both as model systems to study the physics of quasi-zero-dimensional systems and for their device applications. ${ }^{1}$ In particular, the discrete energy level structure of QDs offers significant advantages for modern semiconductor laser technology. In these QD-based devices, nonequilibrium carrier dynamics plays a central role in determining the performance limitations and has, therefore, been subject to considerable research efforts. ${ }^{2,3}$ Most of the studies of carrier relaxation in QDs rely on time-resolved photoluminescence spectroscopy, thus analyzing the scattering of carriers toward the lowest lying QD states. ${ }^{4-8}$ Morover, the relaxation of carriers toward the QD ground states has been analyzed by differential transmission ${ }^{9}$ and $\mathrm{THz}$ absorption spectroscopy. ${ }^{10}$

In contrast, much less is known about the initial steps of the population transfer from the two-dimensional wetting layer (WL) into the zero-dimensional structures. Theoretically, it has been argued that carrier-carrier interaction may be an efficient way to circumvent the "phonon bottleneck" "11,12 that arises from the mismatch of the electronic level spacing with respect to the accessible phonon energies. ${ }^{13}$ This result is supported by the recent experimental observation of ultrafast electron capture in the presence of a $p$-modulation doping within the QDs. ${ }^{14}$

In this letter, we directly analyze the transfer of photoinjected carriers from the WL to fully quantized QD levels. The experiment relies on the transient bleaching of the WL band edge and the QD interband transitions in a two-color femtosecond transmission experiment. For low excitation densities, we find a capture time of 3 ps indicating a very efficient QD filling. Most interestingly, the capture time does not significantly depend on the density of carriers photoin-

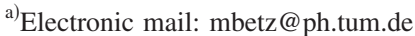

jected into the WL. Moreover, exploiting the selection rules for circularly polarized light, the carrier capture of electrons is shown to be spin preserving.

The nanostructures investigated in this study are selforganized InGaAs QDs grown on a GaAs. The ground-state transitions of the inhomogeneously broadened ensemble are centered at $1.25 \mathrm{eV}$ and the interband energetic spacing of the transitions from different QD shells is approximately 40 $\mathrm{meV}$, i.e., in the order of the longitudinal optical (LO) phonon energy $\hbar \omega_{\mathrm{LO}}=36 \mathrm{meV}$ of the host material GaAs.

The scheme of our experimental approach is displayed in the inset of Fig. 1(a). A pump pulse with a duration of $100 \mathrm{fs}$ is tuned to a central photon energy of $1.51 \mathrm{eV}$ in the absorption continuum of the WL. As a result, electron-hole pairs are generated resonantly in the two-dimensional layer WL and in the GaAs host material. These nonequilibrium carriers experience ultrafast relaxation to the band edge of the WL. Then, the carriers are transfered into fully quantized QD levels. Correspondingly, a transient optical bleaching signal is established reflecting the occupation of the electronic states. These transmission changes of the QD monolayer are detected with a 20 fs broadband probe pulse that is derived from the second branch of a two-color-Ti:Sapphire laser $^{15}$ and spectrally dispersed after transmission through the specimen.

Results for a moderate excitation density and a probe photon energy of $1.45 \mathrm{eV}$, near the band edge of the WL, are shown in Fig. 1(a). The transmission change rises within a few hundred femtoseconds reflecting the thermalization of nonequilibrium photogenerated carriers. More interestingly, the bleaching signal decays exponentially with a time constant of 3 ps (the negative signal detected for delay times $t_{D}>6 \mathrm{ps}$ arises from band-gap renormalization by the nonthermal carriers). These dynamics are accompanied by the buildup of a transmission change probing the QD excited levels [see Fig. 1(b)]. Thus, the present measurement directly 


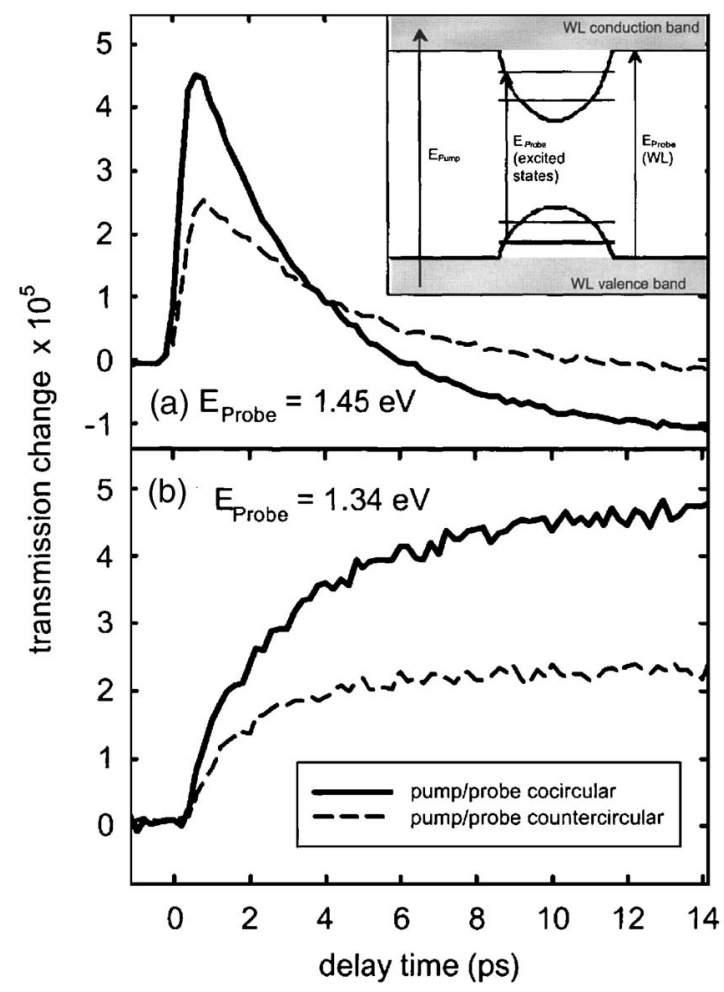

FIG. 1. (a) Transmission changes detected at the band adge of the WL at a probe photon energy of $1.45 \mathrm{eV}$ after excitation with an $100 \mathrm{fs}$ pulse centered at $1.51 \mathrm{eV}$. The solid (dashed) line corresponds to a co-circular (counter-circular) polarization of excitation and probe beams. The inset sketches the bandstructure with the vertical arrows indicating the interband transitions of the study. (b) Corresponding results detecting the transmission changes of the QD excited states at a probe photon energy of $1.34 \mathrm{eV}$.

traces the population transfer from the two-dimensional WL into the QDs.

More detailed insight into the carrier capture process is gained exploiting the spin selectivity of the interband transitions. Circularly polarized excitation yields a preferential spin orientation of the photogenerated carriers. While the spin orientation of holes is rapidly destroyed due to spinorbit interaction, the electron spin in the two-dimensional WL is expected to be preserved over the time scale of the present study. As a consequence, detecting the transmission changes of the WL with a countercircularly polarized probe pulse yields an overall reduction of the transmission change by a factor of 2 [see dashed line in Fig. 1(a)]. The limited degree of polarization may be attributed to the strong disorder and band mixing in the WL. More surprisingly, the nonlinear optical response of the QD interband transitions depends on the polarization configuration in the same manner as observed for the WL [see Fig. 1(b)]. These observations demonstrate that the carrier capture process is a predominantly spin-preserving process. Comparing the degree of polarization for the two probe photon energies, we even do not see evidence for any spin relaxation during the population transfer.

It is interesting to relate this finding to previous studies of spin dynamics in QDs. Several studies ${ }^{16-20}$ report on extraordinary long spin relaxation times for carriers resonantly created inside the QDs. However, these experiments are intrinsically insensitive to the population transfer from the wetting layer into the QDs.
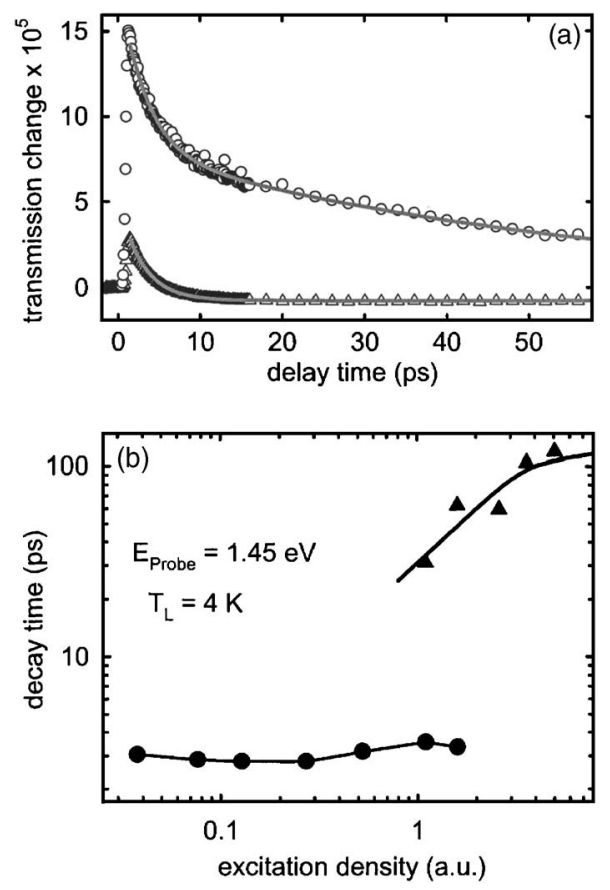

FIG. 2. (a) Transmission changes detected at the band edge of the WL at a probe photon energy of $1.45 \mathrm{eV}$ for two different excitation densities $(0.27$ and 1.6 in the units of part (b)). Symbols: experimental data, gray lines: exponential fits. (b) Decay times of the WL population for various excitation densities. The time constants are extracted from the transmission changes of the WL band edge at $1.45 \mathrm{eV}$ by single and double exponential fits. The abscissa may be interpreted as an estimate for the average quantum dot occupancy, i.e., the average number of electron-hole pairs photogenerated per QD.

In order to identify the nature of the capture process, the QD filling is studied for various excitation densities. To this end, we analyze the transient transmission changes of the WL band edge at a probe photon energy of $1.45 \mathrm{eV}$ as a function of the excitation power. Examples of the experimental data are displayed as symbols in Fig. 2(a). For weak excitation, we find that a exponential decay with a time constant of 3 ps provides a good fit to the experimental data (compare the lower gray line). Most interestingly, the decay times extracted from exponential fits [see filled circles in Fig. 2(b)] do not significantly depend on the excitation density indicating that carrier-carrier scattering has only a minor influence on the capture dynamics.

Increasing the excitation density to values comparable to or larger than the QD density on the sample, the decay of the bleaching signal is effectively slowed down due to the emergence of a slowly varying signal component. This finding is readily understood from the onset of macroscopic state filling effects in the QDs. Modeling these dynamics with a biexponential fit, we identify a fast capture process with a time constant of again 3 ps and a relatively long-lived component [compare the filled triangles in Fig. 2(b)]. For the highest excitation densities of the study, the signal is dominated by massive QD filling and decays only on a time scale approaching typical radiative multiexciton lifetimes in QDs.

The independence of the capture time on the excitation density clearly points toward a phonon-mediated process. The scattering is most likely governed by LO phonons in the polar host material since their energy matches the electronic level spacing of the QDs. It is interesting to relate this finding to previous experimental results claiming the importance 


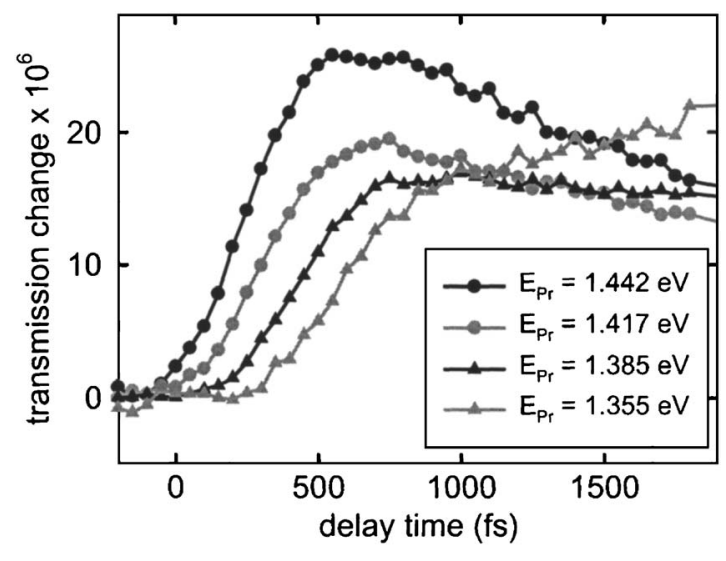

FIG. 3. Transmission changes of the QD monolayer for various probe photon energies $E_{\mathrm{Pr}}$ as indicated in the graph observed for a temperature of $T_{L}=4 \mathrm{~K}$.

of carrier-carrier scattering for the relaxation dynamics. Time-resolved luminescence experiments ${ }^{4,6,7}$ have demonstrated the importance of, e.g., Auger-type processes. However, this experimental approach is intrinsically sensitive to a combination of carrier capture and relaxation toward the QD ground state. Moreover, intraband spectroscopy ${ }^{10}$ has shown a significant influence of electron-hole scattering for the electron dynamics in QDs with a large electronic level spacing as compared to optical phonon energies. In contrast, we study the capture into electronic states near the onset of the WL continuum in QDs with a level spacing comparable to or smaller than the LO phonon energy. In this situation, model calculations $^{13}$ have predicted that phonon-mediated processes may dominate the capture dynamics. Moreover, our results are supported by a time-resolved luminescence study on large InGaAs QDs. ${ }^{8}$ The surprisingly efficient phononmediated relaxation may also be related to the existence of underlying continuum states near the WL band edge ${ }^{21-23}$ that provide an additional relaxation channel. ${ }^{21}$

Additional insight into the energy relaxation of nonthermal carriers in the confined QD states is provided analyzing the transmission changes for various probe photon energies. As displayed in Fig. 3, the transmission change for probe photon energies near the WL band edge (compare the transient for $E_{P r}=1.442 \mathrm{eV}$ ) rises to an maximum within $600 \mathrm{fs}$, as already discussed above. In contrast, the transient transmission changes observed for the QD excited states show a well-resolved delayed onset reflecting the energetic relaxation of carriers through the higher lying confined states of the QDs. This time delay for different probe photon energies allows one to roughly estimate the combined energy relaxation rate of electrons and holes. From the data, we extract a value of one LO phonon energy in a typical time of $150 \mathrm{fs}$ comparable to energy relaxation rates in bulk GaAs. ${ }^{24,25} \mathrm{Ap}-$ parently, the relaxation through the QD states near the continuum of WL states is not strongly hindered by the discrete energy level structure. However, a more detailed analysis of the relaxation dynamics in these QD states near the continuum is difficult since we expect a major influence of the continuous absorption background of the QDs discussed in Refs. 21-23.
In conclusion, we have analyzed the ultrafast spinpreserving population transfer from a two-dimensional WL to QD excited states in a femtosecond transmission experiment. The carrier capture time in our QDs with a level spacing comparable to or smaller than the optical phonon energies amounts to 3 ps and is found to be independent of the excitation density. Taken together, these observations strongly indicate a phonon-mediated process. As a result, we see no significant phonon bottleneck for the filling of the QD states. This finding may be an important ingredient for the optimization of modern QD based devices.

The authors would like to thank P. Gartner, A. Laubereau, and A. Leitenstorfer for valuable discussions. This work has been supported by the Sonderforschungsbereich 631 of the Deutsche Forschungsgemeinschaft.

${ }^{1}$ For an overview, see D. Bimberg, M. Grundmann, and N. N. Ledentsov, Quantum Dot Heterostructures (Wiley, New York, 1998).

${ }^{2}$ P. Borri, W. Langbein, J. M. Hvam, F. Heinrichsdorff, M.-H. Mao, and D. Bimberg, IEEE Photonics Technol. Lett. 12, 594 (2000).

${ }^{3}$ J. Zimmermann, S. T. Cundiff, G. von Plessen, J. Feldmann, M. Arzberger, G. Böhm, M.-C. Amann, and G. Abstreiter, Appl. Phys. Lett. 79, 18 (2001).

${ }^{4}$ B. Ohnesorge, M. Albrecht, J. Oshinowo, A. Forchel, and Y. Arakawa, Phys. Rev. B 54, 11532 (1996).

${ }^{5}$ R. Heitz, M. Veit, N. N. Ledentsov, A. Hoffmann, D. Bimberg, V. M. Ustinov, P. S. Kopev, and Z. I. Alferov, Phys. Rev. B 56, 10435 (1997).

${ }^{6}$ M. De Giorgi, C. Lingk, G. von Plessen, J. Feldmann, S. De Rinaldis, A. Passaseo, M. De Vittorio, R. Cingolani, and M. Lomascolo, Appl. Phys. Lett. 79, 3968 (2001).

${ }^{7}$ D. Morris, N. Perret, and S. Fafard, Appl. Phys. Lett. 75, 3593 (1999).

${ }^{8}$ T. F. Boggess, L. Zhang, G. D. Deppe, D. L. Huffaker, and C. Cao, Appl. Phys. Lett. 78, 276 (2001).

${ }^{9}$ T. S. Sosnowski, T. B. Norris, H. Jiang, J. Singh, K. Kamath, and P. Bhattacharya, Phys. Rev. B 57, R9423 (1998).

${ }^{10}$ T. Müller, F. F. Schrey, G. Strasser, and K. Unterrainer, Appl. Phys. Lett. 83, 3572 (2003).

${ }^{11}$ U. Bockelmann and G. Bastard, Phys. Rev. B 42, 8947 (1990).

${ }^{12}$ H. Benisty, C. M. Sotomayor-Torres, and C. Weisbuch, Phys. Rev. B 44, 10945 (1991).

${ }^{13}$ T. R. Nielsen, P. Gartner, and F. Jahnke, Phys. Rev. B 69, 235314 (2004).

${ }^{14}$ K. Gündogdu, K. C. Hall, T. F. Boggess, G. D. Deppe, and O. B. Shchekin, Appl. Phys. Lett. 85, 4570 (2004).

${ }^{15}$ C. Fürst, A. Leitenstorfer, and A. Laubereau, IEEE J. Sel. Top. Quantum Electron. 2, 473 (1996).

${ }^{16}$ H. Gotoh, H. Ando, H. Kamada, A. Chavez-Pirson, and J. Temmyo, Appl. Phys. Lett. 72, 1341 (1998).

${ }^{17}$ M. Paillard, X. Marie, P. Renucci, T. Amand, A. Jbeli, and J. M. Gerard, Phys. Rev. Lett. 86, 1634 (2001).

${ }^{18}$ A. S. Lenihan, M. V. Gurudev Dutt, D. G. Steel, S. Ghosh, and B. K. Battarcharya, Phys. Rev. Lett. 88, 223601 (2002).

${ }^{19}$ S. Cortez, O. Krebs, S. Laurent, M. Senes, X. Marie, P. Voisin, R. Ferreira, G. Bastard, J.-M. Gerard, and T. Amand, Phys. Rev. Lett. 89, 207401 (2002).

${ }^{20}$ A. I. Tartakovskii, J. Cahill, M. N. Makhonin, D. M. Whittaker, J.-P. R. Wells, A. M. Fox, D. J. Mowbray, M. S. Skolnick, K. M. Groom, M. J. Steer, and M. Hopkinson, Phys. Rev. Lett. 93, 057401 (2004).

${ }^{21}$ Y. Toda, O. Moriwaki, M. Nishioka, and Y. Arakawa, Phys. Rev. Lett. 82, 4114 (1999).

${ }^{22}$ A. Vasanelli, R. Ferreira, and G. Bastard, Phys. Rev. Lett. 89, 216804 (2002).

${ }^{23}$ R. Oulton, J. J. Finley, A. I. Tartakovskii, D. J. Mowbray, M. S. Skolnick, M. Hopkinson, A. Vasanelli, R. Ferreira, and G. Bastard, Phys. Rev. B 68, 235301 (2003).

${ }^{24}$ A. Leitenstorfer, C. Fürst, A. Laubereau, W. Kaiser, G. Tränkle, and G. Weimann, Phys. Rev. Lett. 76, 1545 (1996).

${ }^{25}$ M. Betz, G. Göger, A. Leitenstorfer, K. Ortner, C. R. Becker, G. Böhm, and A. Laubereau, Phys. Rev. B 60, R11265 (1999). 